\title{
HYPEROXIA ENHANCES CEREBRAL INFLAMMATION IN HYPOXIC ISCHEMIC NEWBORN
} MICE

\author{
G. Dohlen ${ }^{1}$, A. Kielland ${ }^{2}$, E.-A. Antal ${ }^{3}$, A. Castellheim ${ }^{3}$, A. Pharo ${ }^{3}$, T.E. Mollnes ${ }^{3}$, E. Thaulow ${ }^{3}$, O.D. \\ Saugstad $^{1}$ \\ ${ }^{1}$ Department of Paediatric Research, University Hospital of Oslo, ${ }^{2}$ University of Oslo, ${ }^{3}$ University Hospital \\ of Oslo, Oslo, Norway
}

Objective: The aim was to study cerebral inflammation after a hypoxic/ischemic cerebral event (HICE) in a newborn luciferase mouse model.

Background: The impact of hyperoxic exposure following HICE is discussed, but may induce inflammation. NF- $\mathrm{kB}$ luciferase mice offer an in vivo reporter model for inflammation.

Methods: HICE was induced by left common carotid artery occlusion followed by hypoxia, in five day old mice. Fifty luciferase mice were randomized to 4 groups: (C) Controls, (RA) HICE followed by room air treatment, (PO) HICE followed by exposure to pure oxygen, (TPO) HICE followed by subcutaneous injection of the antioxidant tempol, before hyperoxia. Head/brain luminescence was measured after one and two days. Cerebral damage was histologically graded on a $0-4$ scale. Thirteen additional mice were randomized to PO or TPO for cytokine analysis.

Results: One day after left sided HICE, luciferase mice exposed to hyperoxia showed significant increase in left hemisphere luminescence $(\mathrm{p}=0,004)$. When tempol was given, this difference disappeared. Histology showed large cerebral injury for both groups exposed to pure oxygen: PO: 3,2 and TPO: 3,4. Cytokine load was reduced by $51 \%$ in the tempol group.

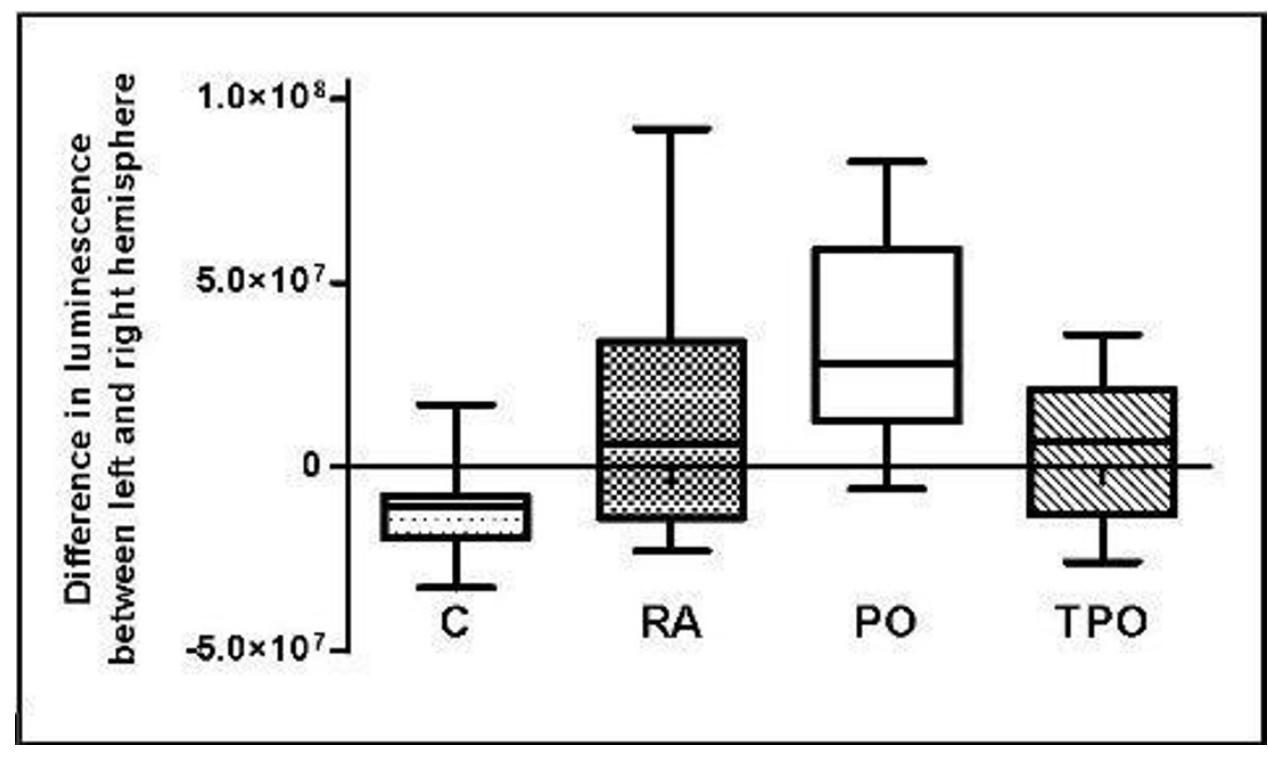

[Luminescence measurements]

Conclusion: HICE followed by exposure to $100 \%$ oxygen increased inflammation and cerebral damage compared to HICE followed by room air. In this study the antioxidant tempol did not protect against cerebral damage, but reduced inflammation. 